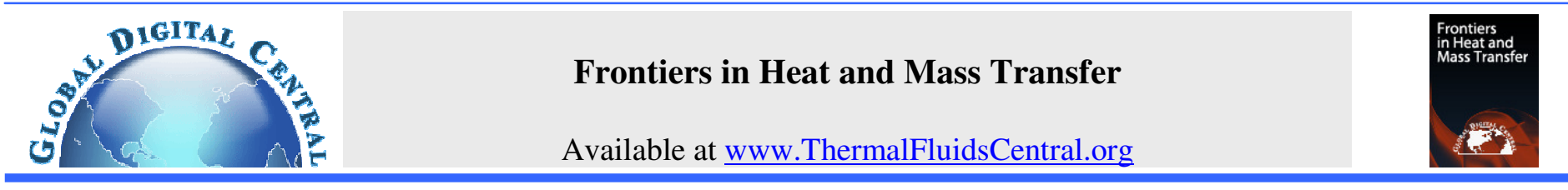

\title{
THERMAL CONDUCTIVITY OF BINARY MIXTURES OF GASES
}

\author{
Etim S. Udoetok \\ Mechanical Engineering Department, University of Uyo, Uyo, Nigeria
}

\begin{abstract}
A model for the coefficient of thermal conductivity of binary mixtures of gases has been derived. The theory presented is based on the assumption of random fluctuations between two possible extreme arrangements of a binary gas mixture. The results obtained from the new model compared favorably with published experimental results. The proposed new model provides a simple approach without sacrificing much accuracy compared to previous models. It is applicable to any binary mixture of gases which includes monoatomic gas mixture, polyatomic gas mixtures and mixtures involving rare gases. The new model can be very useful in analysis like combustion where the main equations are already sophisticated.
\end{abstract}

Keywords: heat, mixture, gas, conduction and resistance.

\section{INTRODUCTION}

Thermal analysis involving gas mixtures usually involves the evaluation of specific heats and thermal conductivities of the gas mixture. While the method for evaluating the specific heat of gas mixture is simple and straight forward, the evaluation of thermal conductivities of gas mixtures have been more sophisticated and several models have been developed (Shapiro, 2004; Li et al., 2011; Lindsay and Bromley, 1950; Mason and Saxena, 1958; Cheung et al., 1962; Simon et al., 1998; Tipton et al., 2009; Papari, 2009). The best approaches are rigorous and poses certain difficulties, so simple predictive models are desirable (Shapiro, 2004). Many thermal systems involves binary mixtures of gases like fuel-oxidizer and carbon dioxidenitrogen, and the binary mixture gas is the simplest mixture level so some works have been focused on it (Simon et al., 1998; Tipton et al., 2009; Imaishi et al., 1984; Song et al., 2010; Barua et al., 1962; Hirschfelder, 1957; Gambhir and Saxena, 1966). There is also a wealth of published experimental data on the conductivity of binary gas mixture systems (Simon et al., 1998; Tipton et al., 2009; Imaishi et al., 1984; Song et al., 2010; Gambhir and Saxena, 1966; Papari et al., 2005; Imaishi and Kestin, 1984; Saxena et al., 1965).

Works on simplified models for thermal conductivities of binary mixtures of gases includes the Wassijewa model which was based on kinetic theory and is given as (Wasiljewa, 1904)

$$
k_{m}=\frac{k_{1}}{1+\Lambda_{12} \frac{x_{2}}{x_{1}}}+\frac{k_{2}}{1+\Lambda_{21} \frac{x_{1}}{x_{2}}}
$$

where the $\Lambda \mathrm{s}$ are based on viscosity and conductivity. An equation of the same form was developed by Sutherland (1895) for the viscosity of gaseous mixtures. The simplified equation by Wassijewa later became complex as the best method for evaluating $\Lambda$ s were investigated (Lindsay and Bromley, 1950; Hirschfelder, 1957). Hirschfelder et al. also proposed a simplified thermal conductivity model which was for gas mixtures as well as for pure gases (Hirschfelder et al., 1948). The Hirschfelder et al equation is a modified form of the Euken equation (Euken, 1913) and is given as

$$
k \cong \mu\left(c_{P}+\frac{5}{4} \frac{R}{M}\right)
$$

The modified Euken equation failed to accurately predict thermal conductivities for gas mixtures. However, it accurately predicted the thermal conductivity of pure gases, since it was derived for polyatomic gas based on contributions due to degrees of freedom so having than one component of gas will amount to a difference in the degrees of freedom (Poling et al., 2000).

Another form of simplified equation was recommended by Kennard (1938) and is given as

$$
k_{m}=k_{1}\left(x_{1}\right)^{2}+K\left(x_{1} x_{2}\right)+k_{2}\left(x_{2}\right)^{2}
$$

The Kennard equation requires the evaluation of a constant $\mathrm{K}$ from experimental work. Lindsay and Bromley (1950) showed that even when $\mathrm{K}$ is determined by least square method, the equation has error of up to $12 \%$. The Kennard equation is more of a curve fit that requires sufficient data from experiment.

Mason and Saxena (1958) later proposed a simplified model given as

$$
k_{\text {mix }}=\sum_{i=1}^{n} k_{i}\left[1+\sum_{k=1, k \neq i}^{n} G_{i k} \frac{x_{k}}{x_{i}}\right]^{-1}
$$

which for the case of a binary gas mixture reduces to the Wassijewa equation form. The Gs are found as a function of molar masses and viscosities.

The purpose of this work is to develop the simplest possible model for the thermal conductivity of a binary mixture of gases that will have an acceptable error or no error at all.

\section{METHOD}

Consider 1-dimensional heat conduction of a mixture of two gases A and $B$ contained between two wall of temperatures $T_{1}$ and $T_{2}$ (see Fig. 1 ). The insulation is used to ensure illustration of heat conduction in $\mathrm{X}$ direction only and allow for the control of mixture component fractions. The following assumptions are used in this analysis:

i. Heat transfer is mainly by conduction and heat transfers by other modes are negligible.

ii. The two gases are perfectly mixed.

*Email: etim.udoetok@yahoo.com 
iii. The gas mixture is a continuum and at the walls, the mixture makes perfect contact with the wall so intermolecular and wall collisions are neglected and expected to reflect in the values of conductivities of the mixture components.

iv. Heat conduction and variation of temperature is in the $\mathrm{x}$-direction only.

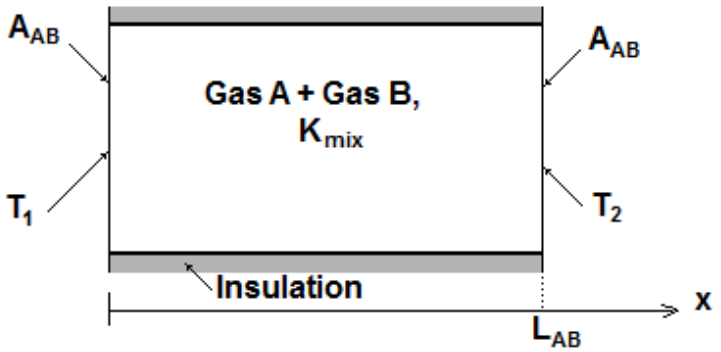

Fig. 1 Mixture of two conducting gases A and B

The molecules of both gases are moving in random motion, so it can be assumed that the gas mixture is fluctuating between two extreme heat conduction arrangements of the gases. These two separate arrangements of the gases are series and parallel arrangement. Therefore, the heat conductivity of the gas mixture can be taken as the average of the heat conductivities obtained for the two heat conduction extremes.

i. Heat conductivity of series arrangement: In this arrangement, the gases are separated and arranged in series as shown in Fig. 2.

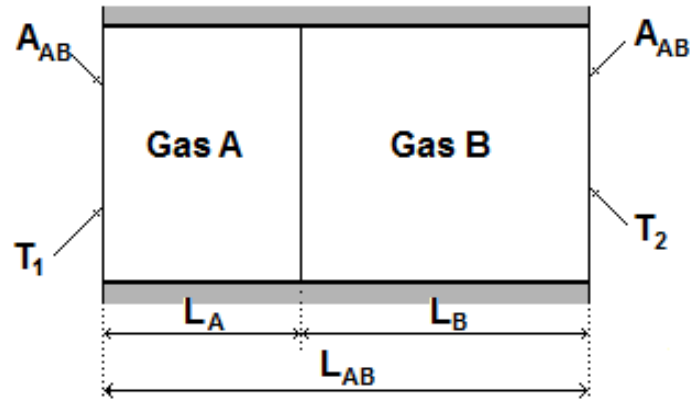

Fig. 2 Separated series arrangement of gases A and B

Thermal resistance method can be used to estimate the overall thermal conductivity of the resulting composite conductor. The overall thermal resistance is the sum of the thermal resistances of the two gases and is give as

$$
\Re_{A-B}=\Re_{A}+\Re_{B}
$$

This implies that

$$
\frac{L_{A B}}{k_{A-B} A_{A B}}=\frac{L_{A}}{k_{A} A_{A B}}+\frac{L_{B}}{k_{B} A_{A B}}
$$

Eq. (6) simplifies to

$$
\frac{1}{k_{A-B}}=\frac{L_{A} A_{A B}}{k_{A} L_{A B} A_{A B}}+\frac{L_{B} A_{A B}}{k_{B} L_{A B} A_{A B}}
$$

It can be seen in Eq. (7) that $L_{A} A_{A B} / L_{A B} A_{A B}$ and $L_{B} A_{A B} / L_{A B} A_{A B}$ represents the mole fractions of $\mathrm{A}$ and $\mathrm{B}$ respectively. Therefore, Eq. (7) becomes

$$
\frac{1}{k_{A-B}}=\frac{X_{A}}{k_{A}}+\frac{X_{B}}{k_{B}}
$$

Thus, the thermal conductivity of the series arrangement is

$$
k_{A-B}=\frac{k_{A} k_{B}}{X_{A} k_{B}+X_{B} k_{A}}
$$

ii. Heat conductivity of parallel arrangement: In this arrangement, the gases are arranged in parallel with the x-coordinate (See Fig. 3). By thermal resistance method, the effective resistance of the parallel composite arrangement is

$$
\frac{1}{\Re_{A \| B}}=\frac{1}{\Re_{A}}+\frac{1}{\Re_{B}}
$$

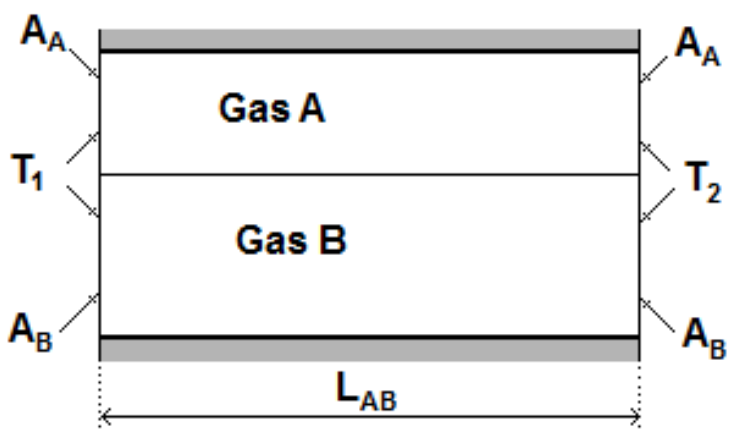

Fig. 3 Separated parallel arrangement of gases A and B

This implies that

$$
\frac{1}{\left(\frac{L_{A B}}{k_{A \| B}\left(A_{A}+A_{B}\right)}\right)}=\frac{1}{\left(\frac{L_{A B}}{k_{A} A_{A}}\right)}+\frac{1}{\left(\frac{L_{A B}}{k_{B} A_{B}}\right)}
$$

Eq. (11) can also be written as

$$
\frac{k_{A \| B}\left(A_{A}+A_{B}\right)}{L_{A B}}=\frac{k_{A} A_{A}}{L_{A B}}+\frac{k_{B} A_{B}}{L_{A B}}
$$

Eq. (12) can be simplified as

$$
k_{A \| B}=\frac{k_{A} A_{A} L_{A B}}{L_{A B}\left(A_{A}+A_{B}\right)}+\frac{k_{B} A_{B} L_{A B}}{L_{A B}\left(A_{A}+A_{B}\right)}
$$

It can be seen in Eq. (13) that $A_{A} L_{A B} /\left(L_{A B}\left(A_{A}+A_{B}\right)\right)$ and $A_{B} L_{A B} /\left(L_{A B}\left(A_{A}+A_{B}\right)\right)$ are the mole fractions of gases $\mathrm{A}$ and $\mathrm{B}$ respectively, thus

$$
k_{A \| B}=k_{A} X_{A}+k_{B} X_{B}
$$

Therefore, the average of the two heat conductivities from Eqs. (9) and (14) gives the mixture heat conductivity.

$$
k_{\text {mix }}=0.5 k_{A-B}+0.5 k_{A \| B}
$$

which implies that

$$
k_{\text {mix }}=0.5 \frac{k_{A} k_{B}}{X_{A} k_{B}+X_{B} k_{A}}+0.5\left(k_{A} X_{A}+k_{B} X_{B}\right)
$$

This new equation, Eq. (16), is a function of mole fractions and thermal conductivities of the pure component gases at the mixture temperature. No special constants or coefficients are involved, except the 0.5 coefficients which were obtained from taking the average between the two heat conductivity extremes.

\section{COMPARISON OF MODEL RESULT WITH PUBLISHED EXPERIMENTAL DATA}

Tables 1-8 shows results of the proposed new model compared with experimental results that have been previously published. The individual gas conductivities were directly taken from the experimental data i.e. $k_{A}=k_{\exp }$ when $X_{A}=1, \quad X_{B}=0 \quad$ and $k_{B}=k_{\exp }$ when $X_{B}=1, X_{A}=0$. The results showed that the proposed new model for estimating thermal conductivities of binary gas mixtures offers results with acceptable degree of accuracy for all cases of binary gas mixtures. High errors of more than $10 \%$ occurred at some combinations of $\mathrm{N}_{2}-\mathrm{He}$ (see Table 2 and 5) and $\mathrm{O}_{2}$ - $\mathrm{He}$ (see Table 4). $\mathrm{N}_{2}$ - $\mathrm{He}$ experimental results from two different authors were checked and similar error by the new model was observed. Therefore, the high error is not directly due to experimental error. However, under the influence of gravity, $\mathrm{He}$ is 
very light and tends to go up or go in the direction opposite the action of gravity when released in a heavier gas. Therefore, the $\mathrm{N}_{2}-\mathrm{He}$ and $\mathrm{O}_{2^{-}}$ $\mathrm{He}$ mixtures have higher tendencies to violate the number two assumption used in the derivation of Eq. (16). In fact, the $\mathrm{N}_{2}-\mathrm{He}$ and $\mathrm{O}_{2}-\mathrm{He}$ mixtures will be perfect mixtures in zero $\mathrm{g}$ or when a mixing device is used to ensure perfect mixing. The errors observed in the $\mathrm{N}_{2^{-}}$ $\mathrm{He}$ and $\mathrm{O}_{2}-\mathrm{He}$ mixture results were due to indirect experimental errors caused by the imperfection of the mixing of the two gases. In order to check the validity of this explanation of the high errors observed in the $\mathrm{N}_{2}-\mathrm{He}$ and $\mathrm{O}_{2}-\mathrm{He}$ results, a mixture of $\mathrm{He}$ and another light gas was checked (see Table 8) and a mixture of $\mathrm{N}_{2}$ and $\mathrm{O}_{2}$ was checked (see Table 6). In both cases it was observed that the new model, Eq. (16), results have errors of less than $5 \%$.

Additionally, more published experimental data were checked and it was observed that the proposed model, Eq. (16) has errors averaging less than $2 \%$ in most cases (Dael and Cauwenbergh, 1968; Ghambir and Saxena, 1966; Imaishi et al., 1984; Imaishi and Kestin, 1984; Lindsay and Bromley, 1950; Mason and Saxena, 1958; Papari et al., 2009; Song et al., 2010; Tipton et al., 2009).

Table 1 Thermal conductivity of NO-CO mixture at various temperatures. $\left(\mathrm{k}\right.$ is in $\left.\mathrm{cal} /{ }^{\circ} \mathrm{C} \cdot \mathrm{cm} \cdot \mathrm{s}\right)$

\begin{tabular}{|c|c|c|c|c|}
\hline Temperature & $\mathrm{X}_{\mathrm{NO}}$ & $\begin{array}{c}\mathrm{k}_{\exp } \times 10^{5} \\
(\text { Barua et al., 1969) }\end{array}$ & $\begin{array}{l}\mathrm{k}_{\text {mix }} \times 10^{5} \\
\text { Eq. }(16) \\
\end{array}$ & $\begin{array}{c}\% \\
\text { Error }\end{array}$ \\
\hline \multirow{7}{*}{$0{ }^{\circ} \mathrm{C}$} & 0.000 & 5.5 & 5.5000 & 0.00 \\
\hline & 0.067 & 5.38 & 5.5073 & 2.37 \\
\hline & 0.246 & 5.44 & 5.5269 & 1.60 \\
\hline & 0.496 & 5.5 & 5.5543 & 0.99 \\
\hline & 0.741 & 5.56 & 5.5813 & 0.38 \\
\hline & 0.959 & 5.6 & 5.6054 & 0.10 \\
\hline & 1.000 & 5.61 & 5.6100 & 0.00 \\
\hline \multirow{7}{*}{$80^{\circ} \mathrm{C}$} & 0.000 & 6.82 & 6.8200 & 0.00 \\
\hline & 0.052 & 6.79 & 6.8363 & 0.68 \\
\hline & 0.246 & 6.88 & 6.8974 & 0.25 \\
\hline & 0.501 & 6.93 & 6.9785 & 0.70 \\
\hline & 0.752 & 7.03 & 7.0593 & 0.42 \\
\hline & 0.949 & 7.19 & 7.1233 & 0.93 \\
\hline & 1.000 & 7.14 & 7.1400 & 0.00 \\
\hline \multirow{7}{*}{$120^{\circ} \mathrm{C}$} & 0.000 & 7.6 & 7.6000 & 0.00 \\
\hline & 0.058 & 7.65 & 7.6046 & 0.59 \\
\hline & 0.273 & 7.7 & 7.6218 & 1.02 \\
\hline & 0.506 & 7.8 & 7.6404 & 2.05 \\
\hline & 0.739 & 7.82 & 7.6590 & 2.06 \\
\hline & 0.948 & 7.83 & 7.6758 & 1.97 \\
\hline & 1.000 & 7.68 & 7.6800 & 0.00 \\
\hline \multirow{6}{*}{$160^{\circ} \mathrm{C}$} & 0.000 & 8.22 & 8.2200 & 0.00 \\
\hline & 0.050 & 8.13 & 8.2274 & 1.20 \\
\hline & 0.499 & 8.44 & 8.2945 & 1.72 \\
\hline & 0.744 & 8.54 & 8.3313 & 2.44 \\
\hline & 0.942 & 8.40 & 8.3612 & 0.46 \\
\hline & 1.000 & 8.37 & 8.3700 & 0.00 \\
\hline \multirow{7}{*}{$200^{\circ} \mathrm{C}$} & 0.000 & 8.61 & 8.6100 & 0.00 \\
\hline & 0.057 & 8.51 & 8.6399 & 1.53 \\
\hline & 0.255 & 8.74 & 8.7446 & 0.05 \\
\hline & 0.504 & 9.12 & 8.8781 & 2.65 \\
\hline & 0.734 & 9.20 & 9.0031 & 2.14 \\
\hline & 0.947 & 9.35 & 9.1205 & 2.45 \\
\hline & 1.000 & 9.15 & 9.1500 & 0.00 \\
\hline
\end{tabular}

Table 2 Thermal conductivity of $\mathrm{N}_{2}$-He mixture at $30^{\circ} \mathrm{C}$ and $45^{\circ} \mathrm{C}$. $\left(\mathrm{k}\right.$ is in $\left.\mathrm{cal} /{ }^{\circ} \mathrm{C} \cdot \mathrm{cm} \cdot \mathrm{s}\right)$

\begin{tabular}{|c|c|c|c|c|}
\hline Temperature & $\mathrm{X}_{\mathrm{He}}$ & $\begin{array}{c}\mathrm{k}_{\exp } \times 10^{5} \\
(\text { Barua, 1959) }\end{array}$ & $\begin{array}{c}\mathrm{k}_{\operatorname{mix}} \times 10^{5} \\
\text { Eq. }(16)\end{array}$ & $\begin{array}{c}\% \\
\text { Error }\end{array}$ \\
\hline \multirow{5}{*}{$30^{\circ} \mathrm{C}$} & 1.0000 & 36.35 & 36.3500 & 0.00 \\
\cline { 2 - 5 } & 0.8864 & 28.43 & 28.0966 & 1.17 \\
\cline { 2 - 5 } & 0.7432 & 21.52 & 22.3014 & 3.63 \\
\cline { 2 - 5 } & 0.6041 & 17 & 18.3364 & 7.86 \\
\cline { 2 - 5 } & 0.4681 & 13.02 & 15.1420 & 16.30 \\
\cline { 2 - 5 } & 0.2893 & 9.904 & 11.4583 & 15.69 \\
\cline { 2 - 5 } & 0.1528 & 8.044 & 8.8711 & 10.28 \\
\cline { 2 - 5 } & 0.0000 & 6.116 & 6.1160 & 0.00 \\
\hline \multirow{5}{*}{${ }^{\circ} \mathrm{C}$} & 1.0000 & 37.64 & 37.6400 & 0.00 \\
\cline { 2 - 5 } & 0.8651 & 28.23 & 28.0441 & 0.66 \\
\cline { 2 - 5 } & 0.7362 & 22.06 & 22.9081 & 3.84 \\
\cline { 2 - 5 } & 0.6241 & 18.08 & 19.5693 & 8.24 \\
\cline { 2 - 5 } & 0.4981 & 14.38 & 16.4179 & 14.17 \\
\cline { 2 - 5 } & 0.2962 & 10.14 & 12.0523 & 18.86 \\
\cline { 2 - 5 } & 0.1562 & 8.337 & 9.2989 & 11.54 \\
\cline { 2 - 5 } & 0.0000 & 6.382 & 6.3820 & 0.00 \\
\hline
\end{tabular}

Table 3 Thermal conductivity of $\mathrm{N}_{2}-\mathrm{Ne}$ mixture at $30{ }^{\circ} \mathrm{C}$ and $45^{\circ} \mathrm{C}$. (k is in $\mathrm{cal} /{ }^{\circ} \mathrm{C} \cdot \mathrm{cm} \cdot \mathrm{s}$ )

\begin{tabular}{|c|c|c|c|c|}
\hline \multirow{3}{*}{ Temperature } & $\mathrm{X}_{\mathrm{Ne}}$ & $\begin{array}{c}\mathrm{k}_{\exp } \times 10^{5} \\
(\text { Barua, 1959) }\end{array}$ & $\begin{array}{c}\mathrm{k}_{\text {mix }} \times 10^{5} \\
\text { Eq. (16) }\end{array}$ & $\begin{array}{c}\% \\
\text { Error }\end{array}$ \\
\hline \multirow{5}{*}{$30{ }^{\circ} \mathrm{C}$} & 1.0000 & 11.62 & 11.6200 & 0.00 \\
\cline { 2 - 5 } & 0.9026 & 10.66 & 10.8806 & 2.07 \\
\cline { 2 - 5 } & 0.7754 & 9.716 & 10.0187 & 3.12 \\
\cline { 2 - 5 } & 0.6954 & 9.914 & 9.5239 & 3.93 \\
\cline { 2 - 5 } & 0.4496 & 7.938 & 8.1692 & 2.91 \\
\cline { 2 - 5 } & 0.3277 & 7.291 & 7.5668 & 3.78 \\
\cline { 2 - 5 } & 0.1286 & 6.524 & 6.6534 & 1.98 \\
\cline { 2 - 5 } & 0.0000 & 6.1 & 6.1000 & 0.00 \\
\hline \multirow{5}{*}{$4{ }^{\circ} \mathrm{C}$} & 1.0000 & 11.98 & 11.9800 & 0.00 \\
\cline { 2 - 5 } & 0.9000 & 10.88 & 11.2175 & 3.10 \\
\cline { 2 - 5 } & 0.7612 & 9.878 & 10.2750 & 4.02 \\
\cline { 2 - 5 } & 0.6504 & 9.365 & 9.5968 & 2.47 \\
\cline { 2 - 5 } & 0.4937 & 8.381 & 8.7221 & 4.07 \\
\cline { 2 - 5 } & 0.3038 & 7.543 & 7.7619 & 2.90 \\
\cline { 2 - 5 } & 0.1480 & 7.032 & 7.0352 & 0.04 \\
\cline { 2 - 5 } & 0.0000 & 6.384 & 6.3840 & 0.00 \\
\hline
\end{tabular}

Table 4 Thermal conductivity of $\mathrm{O}_{2}$-He mixture at $30{ }^{\circ} \mathrm{C}$. $(\mathrm{k}$ is in $\left.\mathrm{cal} /{ }^{\circ} \mathrm{C} \cdot \mathrm{cm} \cdot \mathrm{s}\right)$

\begin{tabular}{|c|c|c|c|c|}
\hline Temperature & $\mathrm{X}_{\mathrm{O} 2}$ & $\begin{array}{c}\mathrm{k}_{\text {exp }} \times 10^{5} \\
(\text { Saxena et al., 1965) }\end{array}$ & $\begin{array}{c}\mathrm{k}_{\text {mix }} \times 10^{5} \\
\text { Eq. (16) }\end{array}$ & $\begin{array}{c}\% \\
\text { Error }\end{array}$ \\
\hline \multirow{5}{*}{$30{ }^{\circ} \mathrm{C}$} & 0.0000 & 36.37 & 36.3700 & 0.00 \\
\cline { 2 - 5 } & 0.1500 & 27 & 26.6563 & 1.27 \\
\cline { 2 - 5 } & 0.3000 & 20.14 & 21.2918 & 5.72 \\
\cline { 2 - 5 } & 0.4500 & 15.64 & 17.3342 & 10.83 \\
\cline { 2 - 5 } & 0.6000 & 12.3 & 14.0070 & 13.88 \\
\cline { 2 - 5 } & 0.7500 & 9.642 & 11.0163 & 14.25 \\
\cline { 2 - 5 } & 0.9000 & 7.621 & 8.2262 & 7.94 \\
\cline { 2 - 5 } & 1.0000 & 6.441 & 6.4410 & 0.00 \\
\hline
\end{tabular}


Table 5 Thermal conductivity of $\mathrm{N}_{2}-\mathrm{He}$ mixture at $30{ }^{\circ} \mathrm{C}$. $\left(\mathrm{k}\right.$ is in cal $/{ }^{\circ}$ $\mathrm{C} \cdot \mathrm{cm} \cdot \mathrm{s})$

\begin{tabular}{|c|c|c|c|c|}
\hline Temperature & $\mathrm{X}_{\mathrm{N} 2}$ & $\begin{array}{c}\mathrm{k}_{\exp } \times 10^{5} \\
(\text { Saxena et } \\
\text { al., 1965) }\end{array}$ & $\begin{array}{c}\mathrm{k}_{\text {mix }} \times 10^{5} \\
\text { Eq. (16) }\end{array}$ & $\begin{array}{c}\% \\
\text { Error }\end{array}$ \\
\hline \multirow{7}{*}{$30{ }^{\circ} \mathrm{C}$} & 0.0000 & 36.5 & 36.5000 & 0.00 \\
\cline { 2 - 5 } & 0.1500 & 26.6 & 26.4152 & 0.69 \\
\cline { 2 - 5 } & 0.3000 & 19.83 & 21.0064 & 5.93 \\
\cline { 2 - 5 } & 0.4500 & 15.34 & 17.0401 & 11.08 \\
\cline { 2 - 5 } & 0.6000 & 11.52 & 13.7057 & 18.97 \\
\cline { 2 - 5 } & 0.7500 & 9.31 & 10.7040 & 14.97 \\
\cline { 2 - 5 } & 0.9000 & 7.401 & 7.8991 & 6.73 \\
\cline { 2 - 5 } & 1.0000 & 6.102 & 6.1020 & 0.00 \\
\hline
\end{tabular}

Table 6 Thermal conductivity of $\mathrm{N}_{2}-\mathrm{O}_{2}$ mixture at $319{ }^{\circ} \mathrm{C}$. $\left(\mathrm{k}\right.$ is in cal/ ${ }^{\circ}$ $\mathrm{C} \cdot \mathrm{cm} \cdot \mathrm{s})$

\begin{tabular}{|c|c|c|c|c|}
\hline Temperature & $\mathrm{X}_{\mathrm{O} 2}$ & $\begin{array}{c}\mathrm{k}_{\text {exp }} \times 10^{5} \\
(\text { Saxena et } \\
\text { al., 1965) }\end{array}$ & $\begin{array}{c}\mathrm{k}_{\text {mix }} \times 10^{5} \\
\text { Eq. (16) }\end{array}$ & $\begin{array}{c}\% \\
\text { Error }\end{array}$ \\
\hline \multirow{3}{*}{$319^{\circ} \mathrm{C}$} & 0.0000 & 10.7 & 10.7000 & 0.00 \\
\cline { 2 - 5 } & 0.6098 & 11.19 & 11.2519 & 0.55 \\
\cline { 2 - 5 } & 1.0000 & 11.62 & 11.6200 & 0.00 \\
\hline
\end{tabular}

Table 7 Thermal conductivity of $\mathrm{SO}_{2}$-Ar mixture at various temperatures. $\left(\mathrm{k}\right.$ is in $\left.\mathrm{cal} /{ }^{\circ} \mathrm{C} \cdot \mathrm{cm} \cdot \mathrm{s}\right)$

\begin{tabular}{|c|c|c|c|c|}
\hline Temperature & $\mathrm{X}_{\mathrm{SO} 2}$ & $\begin{array}{c}\mathrm{k}_{\exp } \times 10^{5} \\
(\mathrm{Gupta}, 1967)\end{array}$ & $\begin{array}{r}\mathrm{k}_{\text {mix }} \times 10^{5} \\
\text { Eq. }(16)\end{array}$ & $\begin{array}{c}\% \\
\text { Error } \\
\end{array}$ \\
\hline \multirow{6}{*}{$39^{\circ} \mathrm{C}$} & 0.000 & 4.389 & 4.3890 & 0.00 \\
\hline & 0.146 & 3.995 & 3.9999 & 0.12 \\
\hline & 0.362 & 3.533 & 3.5085 & 0.69 \\
\hline & 0.572 & 3.228 & 3.0955 & 4.11 \\
\hline & 0.764 & 2.81 & 2.7560 & 1.92 \\
\hline & 1.000 & 2.374 & 2.3740 & 0.00 \\
\hline \multirow{6}{*}{$80.1^{\circ} \mathrm{C}$} & 0.000 & 4.854 & 4.8540 & 0.00 \\
\hline & 0.151 & 4.419 & 4.4806 & 1.39 \\
\hline & 0.28 & 4.233 & 4.1923 & 0.96 \\
\hline & 0.504 & 3.798 & 3.7418 & 1.48 \\
\hline & 0.79 & 3.214 & 3.2330 & 0.59 \\
\hline & 1.000 & 2.893 & 2.8930 & 0.00 \\
\hline \multirow{6}{*}{$121.3^{\circ} \mathrm{C}$} & 0.000 & 5.22 & 5.2200 & 0.00 \\
\hline & 0.166 & 4.964 & 4.8830 & 1.63 \\
\hline & 0.344 & 4.609 & 4.5517 & 1.24 \\
\hline & 0.608 & 4.171 & 4.1044 & 1.60 \\
\hline & 0.798 & 3.838 & 3.8079 & 0.78 \\
\hline & 1.000 & 3.511 & 3.5110 & 0.00 \\
\hline \multirow{6}{*}{$161.1^{\circ} \mathrm{C}$} & 0.000 & 5.699 & 5.6990 & 0.00 \\
\hline & 0.164 & 5.3 & 5.3716 & 1.35 \\
\hline & 0.381 & 4.982 & 4.9732 & 0.18 \\
\hline & 0.556 & 4.684 & 4.6752 & 0.19 \\
\hline & 0.750 & 4.34 & 4.3645 & 0.63 \\
\hline & 1.000 & 3.989 & 3.9890 & 0.00 \\
\hline \multirow{6}{*}{$200.6^{\circ} \mathrm{C}$} & 0.000 & 6.047 & 6.0470 & 0.00 \\
\hline & 0.215 & 5.698 & 5.6809 & 0.30 \\
\hline & 0.373 & 5.479 & 5.4284 & 0.92 \\
\hline & 0.558 & 5.220 & 5.1477 & 1.38 \\
\hline & 0.782 & 4.848 & 4.8264 & 0.45 \\
\hline & 1.000 & 4.530 & 4.5300 & 0.00 \\
\hline
\end{tabular}

Table 8 Thermal conductivity of $\mathrm{H}_{2}$-He mixture at $30{ }^{\circ} \mathrm{C}$. $(\mathrm{k}$ is in $\left.\mathrm{cal} /{ }^{\circ} \mathrm{C} \cdot \mathrm{cm} \cdot \mathrm{s}\right)$

\begin{tabular}{|c|c|c|c|c|}
\hline \multirow{5}{*}{ Temperature } & $\mathrm{X}_{\mathrm{He}}$ & $\begin{array}{c}\mathrm{k}_{\exp } \times 10^{5} \\
\text { Cauwenberg } \\
\text { and Dael, } \\
1971)\end{array}$ & $\begin{array}{c}\mathrm{k}_{\text {mix }} \times 10^{5} \\
\text { Eq. (16) }\end{array}$ & $\begin{array}{c}\% \\
\text { Error }\end{array}$ \\
\hline \multirow{5}{*}{$30{ }^{\circ} \mathrm{C}$} & 0.0000 & 44.380 & 44.3800 & 0.00 \\
\cline { 2 - 5 } & 0.1006 & 42.967 & 43.5306 & 1.31 \\
\cline { 2 - 5 } & 0.2516 & 41.169 & 42.2905 & 2.72 \\
\cline { 2 - 5 } & 0.5066 & 38.502 & 40.2821 & 4.62 \\
\cline { 2 - 5 } & 0.7001 & 37.138 & 38.8221 & 4.53 \\
\cline { 2 - 5 } & 0.8001 & 36.689 & 38.0870 & 3.81 \\
\cline { 2 - 5 } & 0.8523 & 36.590 & 37.7082 & 3.06 \\
\cline { 2 - 5 } & 0.8799 & 36.483 & 37.5093 & 2.81 \\
\cline { 2 - 5 } & 0.8823 & 36.528 & 37.4921 & 2.64 \\
\cline { 2 - 5 } & 0.9133 & 36.535 & 37.2698 & 2.01 \\
\cline { 2 - 5 } & 0.9213 & 36.510 & 37.2126 & 1.92 \\
\cline { 2 - 5 } & 0.9400 & 36.523 & 37.0792 & 1.52 \\
\cline { 2 - 5 } & 0.9600 & 36.563 & 36.9370 & 1.02 \\
\cline { 2 - 5 } & 0.9800 & 36.578 & 36.7953 & 0.59 \\
\cline { 2 - 5 } & 1.0000 & 36.654 & 36.6540 & 0.00 \\
\hline
\end{tabular}

\section{CONCLUSIONS}

The proposed new equation for finding the thermal conductivities of binary mixtures of gases gives results with good degree of accuracy. It is applicable to any binary mixture of gases which includes mixtures of monoatomic, mixtures of polyatomic and mixtures involving rare gases. Unlike other models which are mostly direct functions of mole fractions, component thermal conductivities, viscosities, molar masses, densities and/or temperatures, the proposed new model is just a direct function of mole fractions and component thermal conductivities. The proposed new model still retains high degree of accuracy since thermal conductivities of the mixture components are already functions of viscosity, molar masses and/or temperature as can be seen in Eq. (2) that mainly works for pure gases. It can also be shown from kinetic theory that for pure gases (Turns, 2000)

$$
k=\left(\frac{K_{B}^{3}}{\pi^{3} m \sigma^{4}}\right)^{\frac{1}{2}} T^{\frac{1}{2}}
$$

The new method eliminates some of the complexities involved in models that were previously developed by other authors. However, the new method is not recommended for mixtures involving a high density gas mixed with a very low density gas say, $\mathrm{He}$ or $\mathrm{H}_{2}$, in non-zero gravity conditions.

\section{NOMENCLATURE}

A

area $\left(\mathrm{m}^{2}\right)$

$c_{p} \quad$ specific heat $(\mathrm{J} / \mathrm{kg} \cdot \mathrm{K})$

$G_{i k} \quad$ coefficients base on molar mass and viscosities

$K \quad$ constant $(\mathrm{W} / \mathrm{m} \cdot \mathrm{K})$

$K_{B} \quad$ Boltzmann constant $(\mathrm{J} / \mathrm{K})$

$k_{i} \quad$ thermal conductivity of specie i $(\mathrm{W} / \mathrm{m} \cdot \mathrm{K})$

$L \quad$ length (m)

M molar mass $(\mathrm{kg} / \mathrm{kmol})$

$m \quad$ molecular mass $(\mathrm{kg})$

$R \quad$ gas constant $(\mathrm{kJ} / \mathrm{kg} \cdot \mathrm{K})$

$T \quad$ temperature (K)

$u \quad$ interfacial velocity $(\mathrm{m} / \mathrm{s})$

$X_{i} \quad$ mole fraction of specie i

$x \quad$ coordinate $(\mathrm{m})$

Greek Symbols

$\Lambda_{i j} \quad$ coefficients based on viscosity and conductivity 


\section{$\mathfrak{R}$ thermal resistance $(\mathrm{K} / \mathrm{W})$ \\ $\mu \quad$ viscosity $(\mathrm{kg} / \mathrm{m} \cdot \mathrm{s})$ \\ $\sigma \quad$ molecular diameter (m)}

Subscripts

A $\quad$ gas A

$B \quad$ gas $\mathrm{B}$

$A B \quad$ gas $\mathrm{A}$ and $\mathrm{B}$ mixture

$A-B \quad$ A in series with $\mathrm{B}$

$A \| B \quad$ A in parallel with $\mathrm{B}$

$\exp \quad$ experiment

mix mixture

$1 \quad$ wall 1

$2 \quad$ wall 2

\section{REFERENCES}

Barua, A. K., 1959, "Thermal Conductivity and Eucken Type Correction for Binary Mixtures of N2 with some Rare Gases," Physica, 25, 1275-1286.

http://dx.doi.org/10.1016/0031-8914(59)90049-7

Barua, A. K., Gupta, A. D. and Mukhopadhyay, P., 1969, "Thermal Conductivity of Nitric Oxide, Carbon Monoxide and their Binary Mixtures," International Journal of Heat and Mass Transfer, 12, 587593.

http://dx.doi.org/10.1016/0017-9310(69)90040-4

Cauwenbergh, H. and Dael, W. V., 1971, "Measurements of the Thermal Conductivity of Gases III: Data for Binary Mixture Helium and Argon with Hydrogen Isotopes," Physica, 54, 347-360. http://dx.doi.org/10.1016/0031-8914(71)90182-0

Cheung, H., Bromley, L. A. and Wilke, C. R., 1962, "The Thermal Conductivity of Gas Mixtures," AIChE Journal, 8(2), 221-228. http://dx.doi.org/10.1002/aic.690080219

Dael, V. W. and Cauwenbergh, H., 1968, "Measurements of the Thermal Conductivities of Gases II: Data for Binary Mixtures of He, Ne and Ar," Physica, 40, 173-181.

http://dx.doi.org/10.1016/0031-8914(68)90015-3

Euken, A., 1913, "On the Thermal Conductivity, the Specific Heat and the Viscosity of Gases," Physikalische Zeitschrift, 14, 324-332.

Gambhir, R. S. and Saxena, S. C., 1966, "Thermal Conductivity of Gas Mixtures: Ar-D2, Kr-D2 and Ar-Kr-D2,” Physica, 32, 2037-2043. http://dx.doi.org/10.1016/0031-8914(66)90166-2

Gupta, A. D., 1967, "Thermal Conductivity of Binary Mixtures of Sulphur Dioxide and Inert Gases," International Journal of Heat and Mass Transfer, 10, 921-929.

http://dx.doi.org/10.1016/0017-9310(67)90069-5

Hirschfelder, J. O., Bird, R. B. and Spotz, E. L., 1948, "Transport Properties for Non-Polar Gases," Journal of Chemical Physics, 16(10), 968-980.

http://dx.doi.org/10.1063/1.1746696

Hirschfelder, J. O., 1957, "Thermal Conductivity in Polyatomic Electronically Excited or Chemically Reacting Mixtures III," Sixth International Symposium on Combustion, 351-365.

Imaishi, N., Kestin, J., and Wakeham, W. A., 1984, "Thermal Conductivity of two Binary Mixtures of Gases of Equal Molecular Weight," Physica, 123A, 50-71.

Imaishi, N., and Kestin, J., 1984, “Thermal Conductivity of Methane with Carbon Monoxide,” Physica, 126A, 301-307.
Kennard, E. H., 1938, Kinetic Theory of Gases, McGraw-Hill, New York.

Li, H., Wilhelmsen, O., Lv, Y., Wang, W., and Yan, J., 2011, "Viscosities, Thermal Conductivities and Diffusion Coefficients of CO2 Mixtures: Review of Experimental Data and Theoretical Models," International Journal of Greenhouse Gas Control, 5, 1119-1139. http://dx.doi.org/10.1016/j.ijggc.2011.07.009

Linsay, A. L., and Bromley, L. A., 1950, "Thermal Conductivity of Gas Mixtures," Industrial and Engineering Chemistry, 42(8), 1508-1511. http://dx.doi.org/10.1021/ie50488a017

Mason, E. A., and Saxena, S. C., 1958, "Aproximate Formula for the Thermal Conductivity of Gas Mixtures," The Physics of Fluids, 1(5), 361-369.

http://dx.doi.org/10.1063/1.1724352

Papari, M. M., Mohammad-aghaiee, D., Haghighi, B. and Bousheri, A., 2005, "Transport Properties of Argon-Hydrogen Gaseous Mixture from an Effective Unlike Interaction," Fluid Phase Equilibria, 232, 122-135. http://dx.doi.org/10.1016/j.fluid.2005.03.022

Papari, M. M., Khordad, R., and Akbari, Z., 2009, "Further Property of Lennard-Jones Fluid: Thermal Conductivity," Physica A, 388, 585-592. http://dx.doi.org/10.1016/j.physa.2008.11.003

Poling, B. E., Prausnitz, J. M., and O'Connell, J. P., 2000, The Properties of Gases and Liquids, 5th ed., McGraw-Hill, New York, pp. 10.1-10.70

Saxena, S. C., Saksena, M. P., Gambhir, R. S. and Gandhi, J. M., 1965 "The Thermal Conductivity of Nonpolar Polyatomic Gas Mixtures," Physica, 31, 333-341. http://dx.doi.org/10.1016/0031-8914(65)90038-8

Shapiro, A. A., 2004, "Fluctuation Theory for Transport Properties in Multicomponent Mixtures: Thermodiffusion and Heat Conductivity," Physica A, 332, 151-175.

http://dx.doi.org/10.1016/j.physa.2003.10.014

Simon, J. M., Dysthe, D. K., Fuchs, A. H. and Rousseau, B., 1998, "Thermal Diffusion in Alkane Binary Mixtures: A Molecular Dynamics Approach," Fluid Phase Equilibra, 150-151, 151-159. http://dx.doi.org/10.1016/S0378-3812(98)00286-6

Song, B., Wamg, X., Wu, J. and Liu, Z., 2010, "Prediction of Transport Properties of Pure Noble Gases and some of their Binary Mixtures by ab initio Calculations," Fluid Phase Equilibria, 290, 55-62.

http://dx.doi.org/10.1016/i.fluid.2009.09.010

Sutherland, W., 1895, "The Viscosity of Mixed Gases," Philosophical Magazine Series 5, 40(246), 421-431.

http://dx.doi.org/10.1080/14786449508620789

Tipton, E. L., Tompson, R. V. and Loyalka, S. K., 2009, "ChapmanEnskog Solutions to Arbitrary Order in Sonine Polynomials III: Diffusion, Thermal Diffusion, and Thermal Conductivity in Binary, Rigid-Sphere, Gas Mixture," European Journal of mechanics B/Fluids, 28, 353-386.

http://dx.doi.org/10.1016/j.euromechflu.2008.12.002

Turns, S. R., 2000, An Introduction to Combustion: Concepts and Applications, Second ed., McGraw-Hill, New York, pp. 86-90.

Wassiljewa, A., 1904, "Heat Conduction in Gas Mixtures," Physikalische Zeitschrift, 5 (22), 737-742. 